\title{
Nanoscale
}

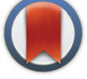

CrossMark

\& click for updates

Cite this: Nanoscale, 2016, 8, 16182

Received 20th June 2016,

Accepted 30th August 2016

DOI: 10.1039/c6nr04959a

www.rsc.org/nanoscale

\section{Electron beam directed etching of hexagonal boron nitride $\uparrow$}

\author{
Christopher Elbadawi, ${ }^{a}$ Trong Toan Tran, ${ }^{a}$ Miroslav Kolíbal, ${ }^{\mathrm{b}, \mathrm{c}}$ Tomáš Šikola, ${ }^{\mathrm{b}, \mathrm{c}}$ \\ John Scott, ${ }^{a}$ Qiran Cai, ${ }^{d}$ Lu Hua Li, ${ }^{d}$ Takashi Taniguchi, ${ }^{e}$ Kenji Watanabe, \\ Milos Toth, ${ }^{* a}$ Igor Aharonovich ${ }^{* a}$ and Charlene Lobo*a
}

Hexagonal boron nitride ( $\mathrm{hBN}$ ) is a wide bandgap van der Waals material with unique optical properties that make it attractive for two dimensional (2D) photonic and optoelectronic devices. However, broad deployment and exploitation of hBN is limited by alack of suitable material and device processing and nano prototyping techniques. Here we present a high resolution, single step electron beam technique for chemical dry etching of hBN. Etching is achieved using $\mathrm{H}_{2} \mathrm{O}$ as a precursor gas, at both room temperature and elevated hBN temperatures. The technique enables damage-free, nano scale, iterative patterning of supported and suspended $2 \mathrm{D} \mathrm{hBN}$, thus opening the door to facile fabrication of hBN-based 2D heterostructures and devices.

Hexagonal BN is a layered material with a wide range of applications in electronics, ${ }^{1}$ photonics ${ }^{2,3}$ and catalysis. ${ }^{4}$ In particular, the two-dimensional form of hexagonal boron nitride (hBN) has recently attracted considerable interest for quantum technologies, ${ }^{5}$ polaritonics, and as a unique natural hyperbolic metamaterial. ${ }^{3,6}$ However, the applicability and wide deployment of hBN in scalable devices is presently limited by a lack of suitable nanoscale processing techniques, particularly growth and etching.

Etching of hBN is typically realized by high temperature annealing in $\mathrm{H}_{2} / \mathrm{Ar}^{7,8}$ or oxidizing environments, ${ }^{9}$ or by reactive ion etching (RIE). ${ }^{10}$ However, the annealing techniques do not allow deterministic fabrication of desired hBN patterns and geometries, and RIE requires cumbersome, multi-step

\footnotetext{
${ }^{a}$ School of Physics and Advanced Materials, University of Technology, Sydney, P.O. Box 123, Broadway, New South Wales 2007, Australia.

E-mail: Milos.Toth@uts.edu.au,Igor.Aharonovich@uts.edu.au, Charlene.Lobo@uts.edu.au

${ }^{b}$ Institute of Physical Engineering, Brno University of Technology, Technicka 2, 61669 Brno, Czech Republic

${ }^{c}$ CEITEC BUT, Brno University of Technology, Technicka 10, 61669 Brno, Czech Republic

${ }^{d}$ Institute of Frontier Materials, Deakin University, Geelong Waurn Ponds Campus, VIC 3216, Australia

${ }^{e}$ National Institute for Materials Science, Ibaraki, 305-0044, Japan

$\dagger$ Electronic supplementary information (ESI) available. See DOI: 10.1039/ c6nr04959a
}

alignment and masking procedures. Direct-write patterning has been demonstrated by high energy electron ${ }^{11-13}$ and ion bombardment ${ }^{10,14}$ using transmission electron microscopy (TEM) and focused ion beam (FIB) techniques. These techniques have limited applicability because the TEM approach requires the use of electron-transparent substrates, and FIB damage compromises surrounding and underlying materials through severe ion beam damage and ion implantation.

Here we present a direct-write, high resolution process for deterministic chemical dry etching of hBN. The etching is performed using a scanning electron microscope (SEM) simply by using $\mathrm{H}_{2} \mathrm{O}$ vapor as an etch precursor gas. The single step process, illustrated schematically in Fig. 1a, is highly versatile, works at room and elevated temperatures, and enables iterative fabrication of arbitrary nano scale patterns defined simply by the scan pattern of the electron beam. The etching can be performed at room temperatures, and at elevated substrate temperatures, consistent with a revised model of gas-mediated electron beam induced etching (EBIE) that was proposed

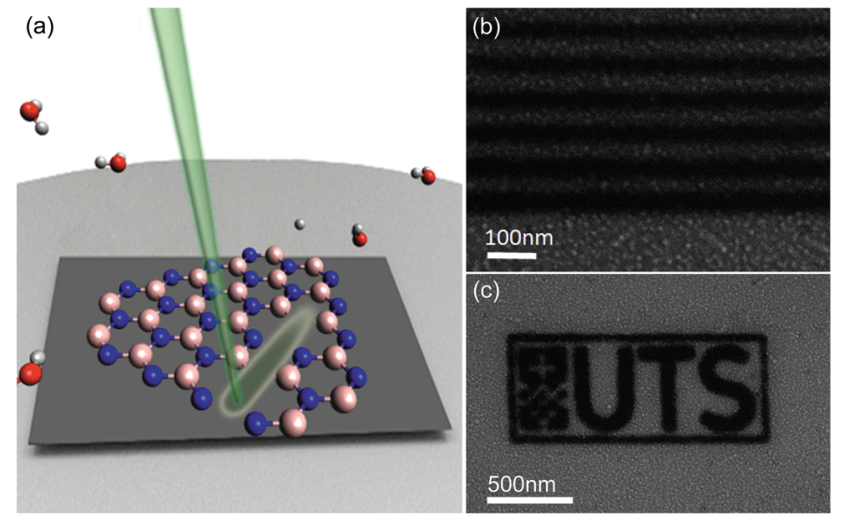

Fig. 1 (a) Schematic illustration of electron beam induced etching of hBN performed using $\mathrm{H}_{2} \mathrm{O}$ vapor as the etch precursor. (b, c) Patterns fabricated in hBN. Dark areas are regions from which hBN was removed by the electron beam. The surface roughness seen in the images is caused by backscattered electrons exiting the sample beyond the region irradiated by the beam. 
recently ${ }^{15}$ in order to explain $\mathrm{H}_{2} \mathrm{O}$-mediated EBIE of single crystal diamond. Our findings expand the toolkit for deterministic nanoscale processing of $\mathrm{hBN}$, and provide independent evidence for a model of $\mathrm{EBIE}^{15}$ in which the critical, rate-limiting role of electrons in EBIE is stimulated desorption of etch reaction products rather than the dissociation of surfaceadsorbed precursor molecules.

Bulk hBN grown by a high temperature, high pressure process ${ }^{16}$ was mechanically exfoliated onto a $\mathrm{Si}(111)$ substrate covered with a native oxide layer. The exfoliated hBN flakes on Si were rinsed with acetone and IPA and dried under flowing $\mathrm{N}_{2}$. To perform EBIE, ${ }^{17-22}$ the hBN samples were mounted onto a heating stage in a customized FEI field emission SEM ${ }^{23}$ and plasma treated overnight at room temperature with a mild $15 \mathrm{~W}$ delocalised plasma in a low vacuum $\mathrm{O}_{2}$ environment of $13 \mathrm{~Pa}$ to remove carbon contaminants resulting from the exfoliation process. The plasma treatment improves EBIE repeatability and enables quantitive measurements of etch rates, ${ }^{24}$ but does not result in etching or damage to the hBN (Fig. S1 $\dagger$ ). Following plasma cleaning, the system was pumped down to high vacuum and the chamber was filled with water vapor to a pressure of $8 \mathrm{~Pa}$ (electron irradiation of hBN in high vacuum gave rise to extremely slow etch rates attributed to the presence of residual $\mathrm{H}_{2} \mathrm{O}$ in the SEM chamber ${ }^{25}$ ). The electron beam current was fixed at $1.35 \mathrm{nA}$ for all EBIE experiments, and the beam energy was either $25 \mathrm{keV}$ (Fig. 3) or $15 \mathrm{keV}$. Etching was done using a focused, Gaussian electron beam, except for etch pits made to characterize the etch rate as a function of time and temperature (Fig. 5), which were fabricated using a stationary top-hat beam with a diameter of $200 \mathrm{~nm}$. Etch pit depths were measured by atomic force microscopy (AFM). Auger electron analysis was performed ex situ using a nano-Auger Electron Spectrometer (AES, Omicron nanoSAM), in ultrahigh vacuum conditions.

Electron beam irradiation in the presence of $\mathrm{H}_{2} \mathrm{O}$ vapor gives rise to localized etching of hBN, and the beam can be scanned to create arbitrary, complex patterns deterministically. As examples, we show an array of waveguides in Fig. 1b, and the logo of the University of Technology Sydney in Fig. 1c. The etch technique is very versatile, but gives rise to the surface roughness seen around the etched patterns. To understand and mitigate this roughening, we characterized the time evolution of the etched surface. Initially (i.e., at low electron fluences), the etch process causes roughening of the hBN surface, followed by complete removal of hBN under the electron beam. The time-evolution of the surface morphology is seen in Fig. 2a where regions 1 to 5 correspond to areas of a hBN flake that were irradiated with electron beam fluences of 1.7, 5.1, 11, 19 and $33 \times 10^{20} \mathrm{~cm}^{-2}$, respectively. Fig. 2b shows Auger electron spectra taken from each of the 5 regions shown in Fig. 2a. Regions 1-4 contain boron and nitrogen, and both elements are absent from region 5 . The spectra also show that the intensity of the $\mathrm{O}^{\mathrm{KLL}}$ oxygen peak increases monotonically with electron fluence, and it is most intense in region 5 . Thus the increase in oxygen is attributed to the native silicon oxide layer of the substrate. The residual fragments seen in regions 1-4 (Fig. 2a) are comprised of boron and nitrogen, as is illustrated by the high resolution secondary electron image, and the boron, nitrogen and oxygen Auger maps shown in Fig. 2c. Raman spectroscopy was also undertaken before and after EBIE of hBN and shows no change to the hBN Raman line (Fig. S2†).

The crystallographic structure of the fragments was confirmed by selected area electron diffraction (SAED). Fig. 3a is a TEM image of a hBN flake that contains a hole (bottom righthand corner) etched by EBIE. Fig. 3b is a SAED pattern taken from a pristine region of the flake that had not been etched by EBIE, while Fig. 3(c and d) shows patterns from two regions indicated on the TEM image, at the periphery of the etch pit. The material in regions (c) and (d) is residue left behind by incomplete etching. All three diffraction patters were indexed as hBN, confirming that the corresponding contrast seen in SEM images (Fig. 1b, c and 2a) corresponds to hBN fragments. Hence, both the Auger and TEM data show that, during the initial phase of EBIE, low dose electron exposure gives rise to the formation of hBN fragments with a diameter of $\sim 30 \mathrm{~nm}$, prior to complete volatilization and removal of the boron and nitrogen constituents.
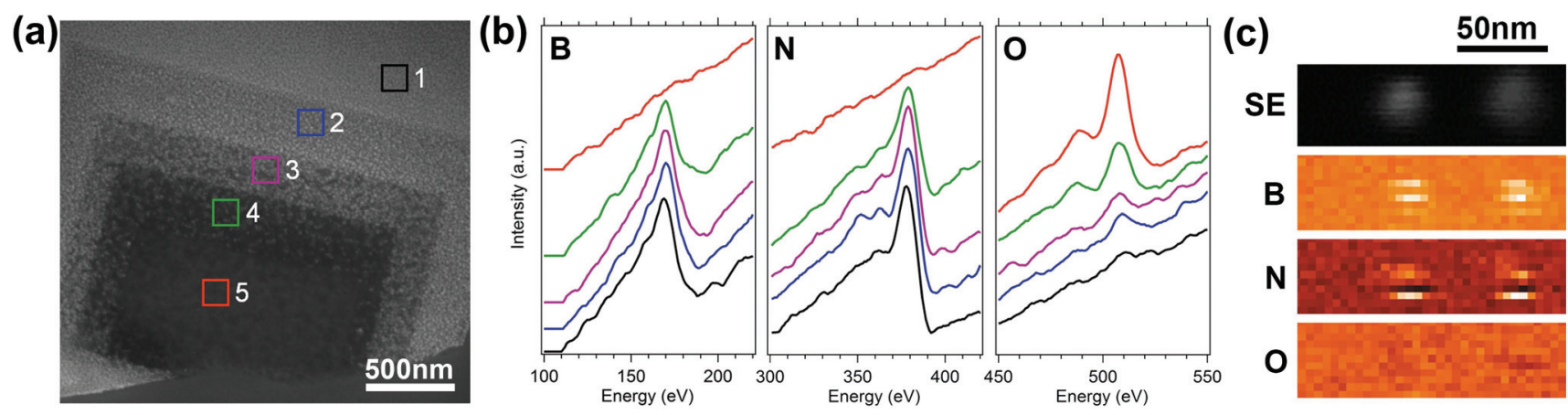

Fig. 2 (a) Flake of hBN that was processed using 5 electron doses corresponding to electron beam fluences of $1.7,5.1,11,19$ and $33 \times 10^{20} \mathrm{~cm}^{-2}$ (regions 1-5, respectively). (b) Auger electron spectra acquired from the 5 regions shown in a. The peaks correspond to the 170 eV $\mathrm{B}^{\mathrm{KLL}}, 379 \mathrm{eV} \mathrm{N}^{\mathrm{KLL}}$ and $507 \mathrm{eV} \mathrm{O} \mathrm{KLL}^{\mathrm{KL}}$ Auger lines. The electron beam energy and current used for Auger analysis were $5 \mathrm{keV} 1 \mathrm{nA}$, respectively. (c) Secondary electron (SE) image and Auger maps of boron nitride fragments generated during etching. 

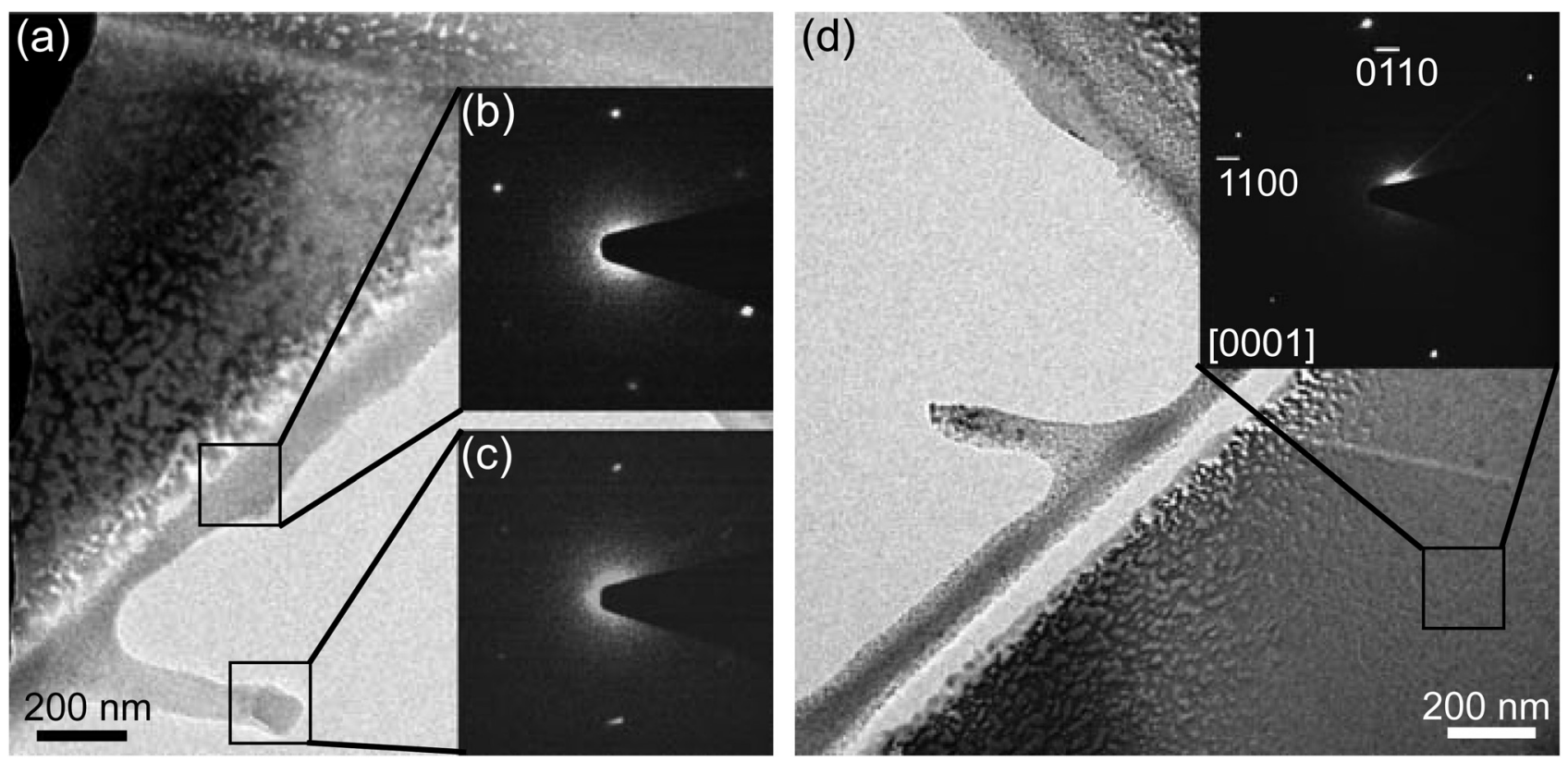

Fig. 3 (a) TEM image of a hBN flake that contains an etch pit (bottom right-hand corner) fabricated by EBIE. The insets (b) and (c) show selected area electron diffraction patterns from the regions indicated on the image. The region indicated in (d) shows a pristine area of the hBN flake and the inset shows its corresponding selected area electron diffraction pattern. All patterns were indexed as hBN.

The hBN fragmentation process is problematic for practical applications of the etch technique because it occurs beyond the immediate area irradiated by the electron beam, and hence damages hBN around the patterned nanostructures (see Fig. $1 \mathrm{~b}$ and c). We attribute this delocalized damage to incomplete etching caused by backscattered electrons (and so-called 'type II' secondary electrons ${ }^{26}$ ) that are emitted from the sample after a large number of scattering events in the bulk substrate below the hBN. The effect can be suppressed simply by minimizing the size of the electron interaction volume in the sample. This can be achieved either by using a low energy electron beam (e.g. $1 \mathrm{keV}$ whereby the width of the electron interaction volume in most materials is on the order of $10 \mathrm{~nm}$ (ref. 27)) or by eliminating the bulk Si substrate. The latter is demonstrated in Fig. 4(a) by a series of lines etched into a hBN flake suspended over a hole in a Si membrane with a $90 \mathrm{~nm}$ thermal oxide layer. Fig. 4(b) is a close-up view of the lines, and clearly shows the presence of fragmented hBN around lines that were etched into hBN residing on the Si membrane (left), and absence of such fragments around regions suspended over a hole (right). The reduction in interaction volume over the hole is illustrated in Fig. $4 \mathrm{c}$ by electron trajectories simulated for a suspended $100 \mathrm{~nm}$ thick flake of hBN (left), and for the same flake residing on a bulk Si substrate (right). The simulations were performed for a $15 \mathrm{keV}$ electron beam using the Monte Carlo package CASINO. ${ }^{28}$ Further SEM images and Monte Carlo simulations showing the differences in fragmentation and surface roughness of suspended and
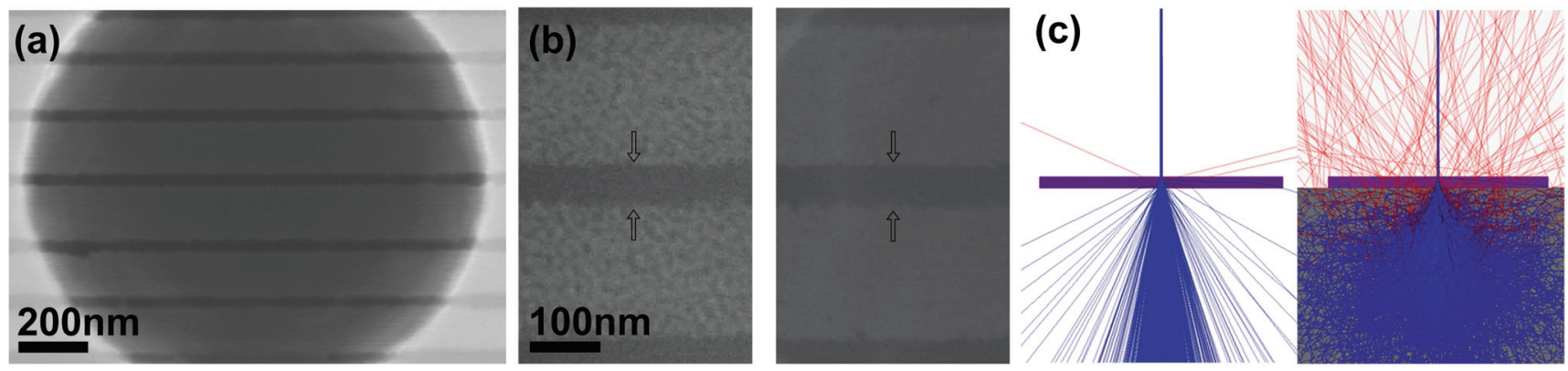

Fig. 4 (a) A series of horizontal lines etched into a hBN flake suspended over a 1.3 micron hole in a Si membrane with a $90 \mathrm{~nm}$ thermal oxide layer. (b) Close-up image of similar lines (indicated by arrows) showing the presence (left) and absence (right) of surface roughness in hBN residing on top of a bulk substrate and over a hole, respectively. (c) Electron trajectories in a suspended $100 \mathrm{~nm}$ multilayer of hBN, and in the same hBN residing on top of a bulk Si substrate. Red lines represent electrons that backscatter out of hBN. The roughness seen in (b) is attributed to backscattered electrons. 
non-suspended hBN at primary beam energies between 5 and $25 \mathrm{keV}$ are shown in Fig. S3-S5. $\dagger$ These results prove that the delocalized damage is indeed caused by backscattered electrons emitted from the sample, and that it can be suppressed simply by minimizing the size of the electron interaction volume.

Having demonstrated the ability to etch hBN, we now characterize the etch process to gain understanding of the mechanism. Etch rate dependencies on temperature ${ }^{15}$ and time $^{24}$ have previously provided key insights into the underlying mechanisms. Hence, Fig. 5 shows plots of etch pit depth versus (a) time and (b) temperature (up to $325{ }^{\circ} \mathrm{C}$ ), revealing that the etch rate is independent of both parameters. The fact that the etch rate does not increase with time indicates that the electron beam does not create chemically active defects below the top monolayer ${ }^{24}$ of hBN. The lack of dependence on temperature implies that the $\mathrm{H}_{2} \mathrm{O}$ precursor molecules dissociate spontaneously, generating chemisorbed species (such as $\mathrm{H}, \mathrm{O}$ and $\mathrm{OH}$ ) that bond to the surface and can contribute to etching, irrespective of the substrate temperature ${ }^{15}$ (up to a temperature of at least $325{ }^{\circ} \mathrm{C}$ ). The dissociation likely takes place at defects or edges of hBN sheets. Preferential chemisorption at defects may explain the fragmentation of hBN flakes (seen in Fig. 2a) that precedes the complete removal of hBN by EBIE.

Our current data do not provide direct evidence for the molecular species of the nitrogen and boron-containing reaction products that desorb from the substrate during etching.
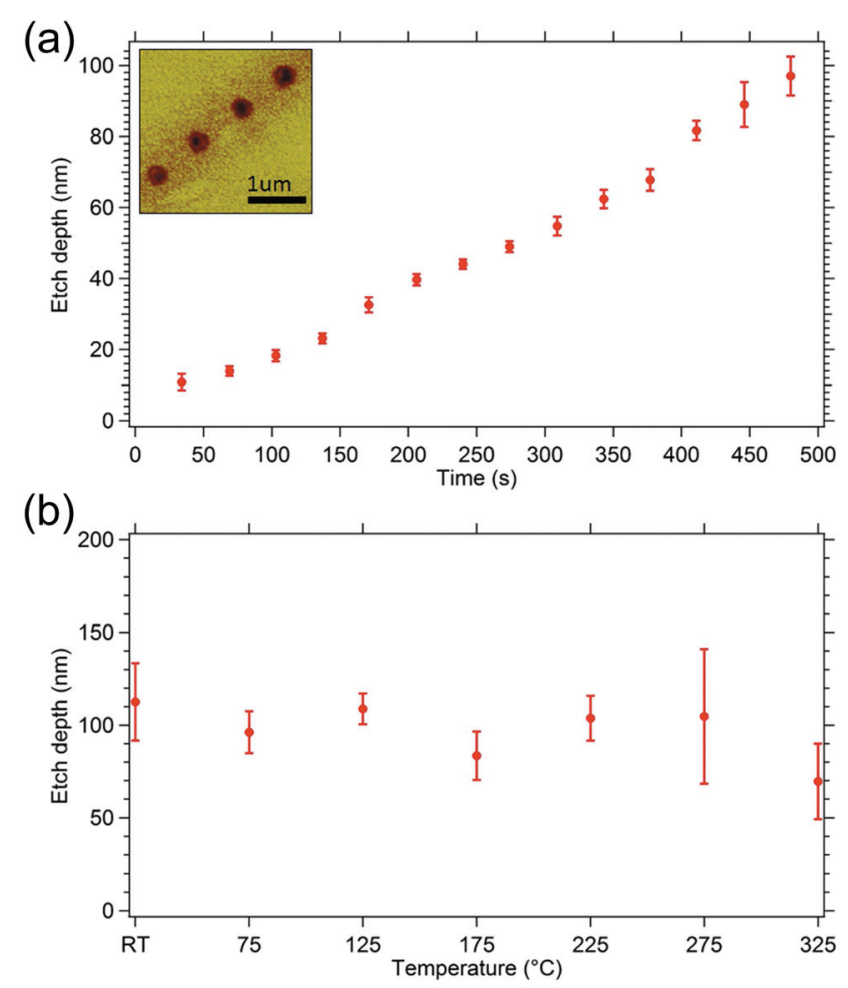

Fig. 5 (a) Etch pit depth plotted as a function of etch time at room temperature. The inset is an AFM image of some of the etch pits used to make the plot. (b) Etch pit depth of hBN versus temperature. The electron beam dwell time was 10 minutes at each temperature.
These molecules are extremely difficult to detect due to the low absolute reaction product generation rates of EBIE. However, we note that the formation of volatile nitrogencontaining molecules is not surprising, as there exist numerous potential candidates such as $\mathrm{N}_{2}, \mathrm{~N}_{2} \mathrm{O}$ and $\mathrm{NO}_{2}$. Potential pathways for volatilization of boron are less obvious and may involve the production of borane $\left(\mathrm{BH}_{3}\right)$ or boric acid $\left(\mathrm{H}_{3} \mathrm{BO}_{3}\right)$.

Finally, we note that the temperature-independence of the etch rate seen in Fig. 5b implies that the critical role of electrons in the etch process is not to dissociate physisorbed precursor molecules (i.e. $\mathrm{H}_{2} \mathrm{O}$ ), as this would result in a rapid, exponential decrease in etch rate with increasing temperature. Instead, a likely key role of the electrons is stimulated desorption of the final reaction products, as has been argued recently for the case of $\mathrm{H}_{2} \mathrm{O}$ EBIE of single crystal diamond. ${ }^{15}$

In conclusion, we have demonstrated a novel $\mathrm{H}_{2} \mathrm{O}$-mediated electron beam induced etching process that enables high resolution patterning of hBN with nanoscale resolution. The etch process likely proceeds through spontaneous dissociative chemisorption of $\mathrm{H}_{2} \mathrm{O}$ at defects and at the edges of hBN sheets, fragmentation of hBN into nanoscale hBN fragments, and volatilization of the nitrogen and boron constituents. The etch rate is found to be temperature independent, indicating the role of the electrons is to remove the final reaction products rather than dissociate the $\mathrm{H}_{2} \mathrm{O}$ precursor molecules. Our results will advance the understanding of hBN chemical stability and accelerate its use in practical optoelectronic and photonic devices.

\section{Acknowledgements}

This work was funded by FEI Company and the Australian Research Council. This work is also supported by European Regional Development Fund (CEITEC - CZ.1.05/1.1.00/ 02.0068) and the Grant Agency of the Czech Republic (Grant No. 15-21581S). M. K. and T. S. acknowledge CF Nano infrastructure for access to the nanoSAM microscope. K. W. and T. T. acknowledge support from the Elemental Strategy Initiative conducted by the MEXT, Japan and a Grant-in-Aid for Scientific Research on Innovative Areas 'Science of Atomic Layers' from JSPS.

\section{References}

1 Y. Lin and J. W. Connell, Nanoscale, 2012, 4, 6908-6939.

2 K. Watanabe, T. Taniguchi and H. Kanda, Nat. Mater., 2004, 3, 404-409.

3 J. D. Caldwell, A. V. Kretinin, Y. Chen, V. Giannini, M. M. Fogler, Y. Francescato, C. T. Ellis, J. G. Tischler, C. R. Woods, A. J. Giles, et al., Nat. Commun., 2014, 5, 5221.

4 K. Uosaki, G. Elumalai, H. Noguchi, T. Masuda, A. Lyalin, A. Nakayama and T. Taketsugu, J. Am. Chem. Soc., 2014, 136, 6542-6545.

5 T. T. Tran, K. Bray, M. J. Ford, M. Toth and I. Aharonovich, Nat. Nanotechnol., 2015, 11, 37-41. 
6 S. Dai, Z. Fei, Q. Ma, A. Rodin, M. Wagner, A. McLeod, M. Liu, W. Gannett, W. Regan and K. Watanabe, Science, 2014, 343, 1125-1129.

7 L. Wang, B. Wu, L. Jiang, J. Chen, Y. Li, W. Guo, P. Hu and Y. Liu, Adv. Mater., 2015, 27, 4948-4948.

8 S. Sharma, G. Kalita, R. Vishwakarma, Z. Zulkifli and M. Tanemura, Sci. Rep., 2015, 5, 10426-10426.

9 Y. Liao, K. Tu, X. Han, L. Hu, J. W. Connell, Z. Chen and Y. Lin, Sci. Rep., 2015, 5, 14510.

10 Z. Liu, L. Ma, G. Shi, W. Zhou, Y. Gong, S. Lei, X. Yang, J. Zhang, J. Yu, K. P. Hackenberg, A. Babakhani, J.-C. Idrobo, R. Vajtai, J. Lou and P. M. Ajayan, Nat. Nanotechnol., 2013, 8, 119-124.

11 J. C. Meyer, A. Chuvilin, G. Algara-Siller, J. Biskupek and U. Kaiser, Nano Lett., 2009, 9, 2683-2689.

12 L. Sun, F. Banhart and J. Warner, MRS Bull., 2015, 40, 29-37.

13 G. H. Ryu, H. J. Park, J. Ryou, J. Park, J. Lee, G. Kim, H. S. Shin, C. W. Bielawski, R. S. Ruoff, S. Hong and Z. Lee, Nanoscale, 2015, 7, 10600-10605.

14 S. Linas, R. Fulcrand, F. Cauwet, B. Poinsot and A. Brioude, RSC Adv., 2015, 5, 49231-49234.

15 A. A. Martin, A. Bahm, J. Bishop, I. Aharonovich and M. Toth, Phys. Rev. Lett., 2015, 115, 255501-255501.

16 T. Taniguchi and K. Watanabe, J. Cryst. Growth, 2007, 303, 525-529.
17 I. Utke, S. Moshkalev and P. Russell, Nanofabrication using focused ion and electron beams: principles and applications, Oxford University Press, 2012.

18 M. Toth, Appl. Phys. A: Solids Surf., 2014, 117, 1623-1629.

19 C. R. Arumainayagam, H.-L. Lee, R. B. Nelson, D. R. Haines and R. P. Gunawardane, Surf. Sci. Rep., 2010, 65, 1-44.

20 I. Utke, P. Hoffmann and J. Melngailis, J. Vac. Sci. Technol., $B, 2008,26,1197-1276$.

21 S. J. Randolph, J. D. Fowlkes and P. D. Rack, Crit. Rev. Solid State Mater. Sci., 2006, 31, 55-89.

22 I. Utke and A. Goelzhaeuser, Angew. Chem., Int. Ed., 2010, 49, 9328-9330.

23 M. Toth, C. J. Lobo, W. R. Knowles, M. R. Phillips, M. T. Postek and A. E. Vladar, Nano Lett., 2007, 7, 525-530.

24 A. A. Martin, M. R. Phillips and M. Toth, ACS Appl. Mater. Interfaces, 2013, 5, 8002-8007.

25 A. A. Martin, G. McCredie and M. Toth, Appl. Phys. Lett., 2015, 107, 041603.

26 J. Goldstein, D. E. Newbury, P. Echlin, D. C. Joy, A. D. Romig Jr., C. E. Lyman, C. Fiori and E. Lifshin, Scanning electron microscopy and X-ray microanalysis: a text for biologists, materials scientists, and geologists, Springer Science \& Business Media, 2012.

27 D. Joy and S. Luo, Scanning, 1989, 11, 176-180.

28 P. Hovington, D. Drouin and R. Gauvin, Scanning, 1997, 19, 1-14. 